\title{
HIV-positive kidney transplants for HIV-positive individuals: Attitudes and concerns of South African patients and health care workers
}

\author{
Suzanne Gokool, June Fabian, W D Francois Venter, Catherine MacPhail, Saraladevi Naicker
}

To the Editor: In South Africa, an estimated $30 \%$ of the cadaveric donor pool is HIV-infected; in consequence, these organs are discarded. An undersupply of donor organs combined with limited resources tends to exclude HIV-positive patients from renal replacement programmes. We evaluated the acceptance of using HIV-positive donor kidneys for transplantation into HIV-infected recipients, and found that the vast majority $(90 \%$ of health care workers and $80 \%$ of patients, $N=20$ and 80 , respectively) found this approach acceptable for expanding the organ donor pool, which indicates broad patient and health care worker support for using HIV-infected donor kidneys.

\section{Background}

South Africa has one of the largest HIV epidemics and largest antiretroviral (ARV) access programmes in the world. ${ }^{1}$ With ARV therapy extending life expectancy, management of HIVinfected individuals is increasingly focusing on age-, HIV- and ARV treatment-related co-morbidities. ${ }^{2} \mathrm{~A}$ serious co-morbidity is HIV-related end-stage kidney disease (ESKD), which has greatly increased the burden of chronic kidney disease (CKD) in South Africa - a morbidity for which the therapeutic infrastructure is already ill-equipped to cope with the renal complications caused by common conditions such as diabetes and hypertension. ${ }^{3}$ South Africa is, however, one of the few African countries to have private and state-funded dialysis and transplant programmes.

Comparative studies of treatment options for ESKD have shown that transplantation rather than dialysis is the most cost-effective form of treatment, greatly improving patients'

Department of Infectious and Tropical Diseases, London School of Hygiene and Tropical Medicine, London

Suzanne Gokool, BSc, MSc, PhD

Division of Nephrology, Charlotte Maxeke Johannesburg Hospital and University of the Witwatersrand, Johannesburg

June Fabian, FCP (SA), Cert Neph (SA)

Saraladevi Naicker, PhD, FRCP (Lond)

Reproductive Health and HIV Research Unit, University of the Witwatersrand, Johannesburg

W D Francois Venter, FCP (SA), DTM\&H, Dip HIV Management Catherine MacPhail, $\mathrm{PhD}$

Corresponding author: J Fabian (june.fabian@mweb.co.za) quality of life and survival. ${ }^{4,5} \mathrm{HIV}$-positive patients were previously excluded from renal replacement therapy owing to poor outcomes, but evidence indicates that transplantation in selected HIV-infected patients can be beneficial, as 1- and 2-year survival rates were higher than of those maintained on dialysis. ${ }^{6}$ In addition, transplantation using both kidneys from the same donor for separate HIV-infected and -uninfected patients demonstrated that graft and patient survival were comparable in both recipient groups after 5 years. ${ }^{7}$

South African health policy guidelines have therefore been revised to allow HIV-infected patients access to dialysis and kidney transplantation, provided that they meet the eligibility criteria: a CD4 count $>200$ cells $/ \mu 1$; an undetectable HIV viral load; and adherence to a stable ARV regimen. ${ }^{8}$ Unfortunately, most HIV-infected and uninfected patients are not offered access to chronic dialysis or transplantation owing to a shortage of dialysis slots and organ donors. A recent proposed solution is to use HIV-positive cadaveric donor kidneys for transplantation into HIV-infected recipients. ${ }^{9}$ The potential hazards surrounding this procedure are presently unquantifiable, but may include the transfer of resistant HIV strains or opportunistic infections, and transplantation of a kidney with undiagnosed HIV-CKD. The attitudes, concerns and acceptability of using HIV-positive kidneys for transplantation into HIV-positive recipients were assessed by interviewing HIV-infected and uninfected individuals, with and without renal disease, and health care workers.

\section{Methods}

Study participants fulfilling the transplant programme inclusion criteria, as well as those without kidney disease, were recruited from the appropriate clinics by convenience sampling ( $N=20$ for each group) at Charlotte Maxeke Johannesburg, Helen Joseph and Chris Hani Baragwanath hospitals in Johannesburg. They were placed into 4 groups: group 1 - HIV-infected individuals on ARVs without identified kidney disease; group 2 - HIV-infected individuals on ARVs with established stages $(1-5)$ of $C K D$, including those on dialysis; group 3 - HIV-uninfected individuals on dialysis; and group 4 - HIV-uninfected individuals with a functioning graft following transplant. Half of the total number of interviewees were HIV-positive, and 75\% suffered from CKD. An additional group comprising health care workers in the field of kidney disease and HIV was interviewed using a scenario-based questionnaire. After invitation to participate and providing 
signed, informed consent, questionnaire-based interviews were conducted in the participants' chosen indigenous language with the aid of a translator. Univariate comparisons were tested using the $\chi^{2}$ test, and all $p$-values are reported as two-sided with a significance value at $p<0.05$ (STATA 10).

\section{Results}

\section{Attitudes of patients}

Table I shows the demographic and clinical characteristics of the study groups. All participants were asked whether they thought that people with HIV infection should receive kidney transplants, and whether they would accept an HIV-positive kidney. If the answer to the latter question was ' $\mathrm{No}^{\prime}$, they were asked whether other individuals on ARVs should have access to kidney transplants. Most respondents in all groups thought access should be granted to HIV-positive people, with the highest proportion of affirmative responses (90\% and 95\%) coming from the two HIV-positive groups. The responses from these groups were not statistically different $(p=0.077)$. HIV-positive patients were more likely to say that they would accept an HIV-positive kidney graft $(p<0.001)$; while a minority of HIV-negative patients would accept one. All groups thought that HIV-positive individuals on stable ARVs should be offered HIV-positive kidneys; the highest positive responses came

Table I. Demographic and clinical characteristics of study participants grouped according to HIV status and the presence of end-stage kidney disease

\begin{tabular}{|c|c|c|c|c|c|}
\hline & $\begin{array}{l}\text { Group } 1 \\
N=20\end{array}$ & $\begin{array}{l}\text { Group } 2 \\
N=20\end{array}$ & $\begin{array}{l}\text { Group } 3 \\
N=20\end{array}$ & $\begin{array}{l}\text { Group } 4 \\
N=20\end{array}$ & $\begin{array}{l}\text { Overall } \\
\%\end{array}$ \\
\hline \multicolumn{6}{|c|}{ Demographic characteristics } \\
\hline \multicolumn{6}{|l|}{ Age group (years) (\%) } \\
\hline $19-29$ & 20 & 15 & 20 & 25 & 20.0 \\
\hline $30-39$ & 60 & 35 & 30 & 15 & 35.0 \\
\hline $40-49$ & 10 & 40 & 30 & 35 & 28.8 \\
\hline $50+$ & 10 & 10 & 20 & 25 & 16.2 \\
\hline \multicolumn{6}{|l|}{ Age (years) } \\
\hline Median & 34.5 & 39.0 & 39.5 & 42.5 & 38.0 \\
\hline $\mathrm{IQR}$ & $30-39$ & $34-46$ & $31-47$ & $30-50$ & $31-45$ \\
\hline \multicolumn{6}{|l|}{ Gender (\%) } \\
\hline Male & 25 & 55 & 60 & 55 & 48.8 \\
\hline \multicolumn{6}{|l|}{ Ethnicity $(\%)$} \\
\hline Black & 95 & 100 & 75 & 60 & 82.5 \\
\hline White & 5 & 0 & 15 & 20 & 10.0 \\
\hline Coloured & 0 & 0 & 0 & 15 & 3.8 \\
\hline Indian & 0 & 0 & 10 & 5 & 3.7 \\
\hline \multicolumn{6}{|c|}{ Clinical HIV-related information } \\
\hline \multicolumn{6}{|c|}{ Length of time on ARVs } \\
\hline Median (months) & 36 & 38 & $\mathrm{~N} / \mathrm{A}$ & $\mathrm{N} / \mathrm{A}$ & 36 \\
\hline $\mathrm{IQR}$ & $30-46$ & $27-48$ & & & $30-48$ \\
\hline \multicolumn{6}{|l|}{ Baseline CD4 count, } \\
\hline Median (cells/ $\mu \mathrm{l})$ & 91.5 & 137 & N/A & N/A & 99 \\
\hline $\mathrm{IQR}$ & $33-150$ & $63-259$ & & & $45-194$ \\
\hline \multicolumn{6}{|l|}{ Current CD4 count, } \\
\hline Median (cells/ $\mu \mathrm{l})$ & 450 & 383 & $\mathrm{~N} / \mathrm{A}$ & $\mathrm{N} / \mathrm{A}$ & 418 \\
\hline IQR & $368-595$ & $310-479$ & & & $340-516$ \\
\hline \multicolumn{6}{|c|}{ Clinical renal disease-related information } \\
\hline \multicolumn{6}{|c|}{ Reason for kidney failure (\%) } \\
\hline CKD & N/A & 10 & 20 & 15 & 15.0 \\
\hline Diabetes & N/A & 10 & 5 & 5 & 6.7 \\
\hline Hypertension & $\mathrm{N} / \mathrm{A}$ & 50 & 60 & 55 & 55.0 \\
\hline Other & $\mathrm{N} / \mathrm{A}$ & 30 & 15 & 25 & 23.3 \\
\hline
\end{tabular}


from the HIV-uninfected groups 3 and 4 (75\% and 83.3\% respectively). HIV-infected participants were specifically asked whether they would accept kidneys from family members, whether HIV-infected or uninfected. The majority stated that they would accept kidneys from infected and uninfected family members.

Forty-five per cent of HIV-infected participants without kidney disease stated that they would choose transplantation as the preferred method of renal replacement therapy; $25 \%$ would consider haemodialysis, peritoneal dialysis or transplantation. When asked if they would be willing to consider dialysis, $55 \%$ replied that they would, with an additional $20 \%$ noting that they would consider dialysis if they could afford it. Twenty-five per cent of participants said they would refuse dialysis as a treatment option, the most common $(30 \%)$ reason for refusal among them being the time and high travel cost involved.

\section{Attitudes of health care workers}

Health care workers $(N=20)$, who were predominantly nurses (55\%), in renal units and ARV clinics were given clinical scenario-based questionnaires. Ninety per cent agreed that HIV-positive individuals should be provided with kidney transplants if patients met the proposed eligibility criteria. However, the need for education of the public to encourage organ donations (15\%) and full information about transplantation (10\%) was respectively noted by health care workers.

\section{Discussion and conclusion}

Our results suggest that both patients and health care workers find it acceptable to include HIV-positive cadaveric donors for transplantation into eligible HIV-positive recipients, despite the unknown risks. The key reasons given for providing transplants to HIV-positive individuals using HIV-positive kidneys were to afford them the opportunity for longer survival, to avoid discrimination because of their HIV status, and to allow them equal rights of health care access compared with HIV-negative patients. Our findings suggest that, unless clear biological risks in using HIV-positive grafts are identified, providing patients with full information about uncertainties and risks will allow them to make a decision on transplantation with HIV-positive kidneys. Such an approach would dramatically increase the donor pool of available kidneys. At the time of writing, 4 HIV-infected donor kidneys were successfully transplanted into HIV-positive recipients in South Africa. ${ }^{10}$ Research should now focus on graft survival and careful follow-up of recipients to assess short-, mediumand long-term risks associated with this new procedure.

The authors thank Ms Lucie Ndlovu for her assistance with translation and subject recruitment; Drs T Gerntholtz and M Radev for facilitating access to participants at Chris Hani Baragwanath and Helen Joseph hospitals, respectively; and the participants in this study. The study was funded in part by the Trust Fund of the London School of Hygiene and Tropical Medicine. Approval for the study was obtained from the Human Research Ethics Committee of the University of the Witwatersrand and the London School of Hygiene and Tropical Medicine (reference numbers M080502 and 07/342 respectively).

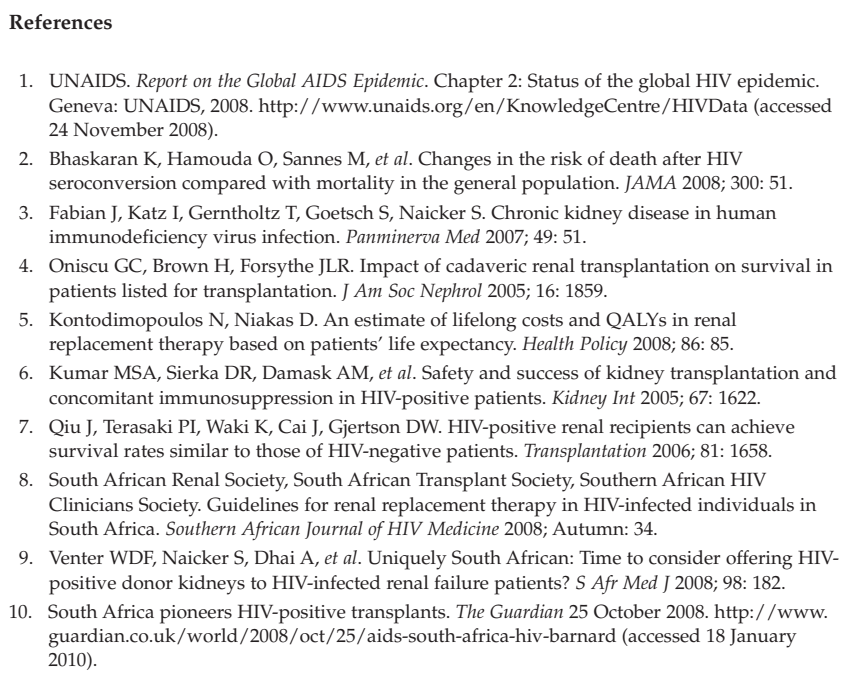

1. UNAIDS. Report on the Global AIDS Epidemic. Chapter 2: Status of the global HIV epidemic. Geneva: UNAIDS, 2008. http://www.unaids.org/en/KnowledgeCentre/HIVData (accessed 24 November 2008).

2. Bhaskaran K, Hamouda O, Sannes M, et al. Changes in the risk of death after HIV seroconversion compared with mortality in the general population. JAMA 2008; 300: 51

3. Fabian J, Katz I, Gerntholtz T, Goetsch S, Naicker S. Chronic kidney disease in human Fabian J, Katz I, Gerntholtz T, Goetsch S, Naicker S. Chronic kide
immunodeficiency virus infection. Panminerva Med 2007; 49: 51.

4. Oniscu GC, Brown H, Forsythe JLR. Impact of cadaveric renal transplantation on survival in patients listed for transplantation. J Am Soc Nephrol 2005; 16: 1859.

5. Kontodimopoulos N, Niakas D. An estimate of lifelong costs and QALYs in renal replacement therapy based on patients' life expectancy. Health Policy 2008; 86: 85.

6. Kumar MSA, Sierka DR, Damask AM, et al. Safety and success of kidney transplantation and concomitant immunosuppression in HIV-positive patients. Kidney Int 2005; 67: 1622.

7. Qiu J, Terasaki PI, Waki K, Cai J, Gjertson DW. HIV-positive renal recipients can achieve survival rates similar to those of HIV-negative patients. Transplantation 2006; 81: 1658 .
surve 8. South African Renal Society, South African Transplant Society, Southern African HIV Clinicians Society. Guidelines for renal replacement therapy in HIV-infected individuals in South Africa. Southern African Journal of HIV Medicine 2008; Autumn: 34

9. Venter WDF, Naicker S, Dhai A, et al. Uniquely South African: Time to consider offering HIVpositive donor kidneys to HIV-infected renal failure patients? S Afr Med J 2008; $98: 182$.

10. South Africa pioneers HIV-positive transplants. The Guardian 25 October 2008. http:// www guardian.co.uk/world/2008/oct/25/aids-south-africa-hiv-barnard (accessed 18 January 2010).

Accepted 11 September 2009. 